\title{
Left-right asymmetry in firing rate of extra-retinal photosensitive neurons in the crayfish
}

\author{
José A. Pacheco-Ortiz ${ }^{1}$, Juan C. Sánchez-Hernández ${ }^{1}$, Leonardo Rodríguez-Sosa², Gabina \\ Calderón-Rosete ${ }^{2}$ and Edgar Villagran-Vargas ${ }^{3}$ \\ ${ }^{1}$ Faculty of Science, Autonomous University of the State of Mexico, Mexico City, Mexico \\ ${ }^{2}$ Physiology Department, Faculty of Medicine, National Autonomous University of Mexico, Mexico City, Mexico \\ ${ }^{3}$ University of De La Salle Bajio, Mexico
}

\begin{abstract}
The purpose of this paper is to explore the firing rate of the caudal photoreceptors (CPRs) from the sixth abdominal ganglion of the crayfish Cherax quadricarinatus. We use simultaneous extracellular recordings on left and right CPR in the isolated ganglion $(n=10)$. The CPRs showed an asymmetry in the spontaneous activity and light-induced response. In darkness, we observed one subgroup (70\%) in which the left CPR (CPR-L) and right CPR (CPR-R) had spontaneous firing rates with a median of $18 \mathrm{impulses} / \mathrm{s}$ and $6 \mathrm{impulses} / \mathrm{s}$, respectively. In another subgroup (20\%), the CPR-R had a median of 15 impulses/s and the CPR-L had 8 impulses/s. In both groups, the differences were significant. Furthermore, the CPRs showed an asymmetrical photoresponse induced by a pulse of white light (700 Lux, 4 s). In one subgroup (30\%), the CPR-L showed light-induced activity with a median of $73 \%$, (interquartile range, $I Q R=51$ ), while the $C P R-R$ had a median of $41 \%$, (IQR $=47)$. In another subgroup $(70 \%)$, the CPR-R showed a median of $56 \%,(\mathrm{IQR}=51)$ and the CPR-L had a median of $42 \%$, (IQR $=46)$. In both groups, the differences were significant. Moreover, we observed a differential effect of temperature on CPR activity. These results suggest a functional asymmetry in both activities from left and right CPRs. These CPR activity fluctuations may modulate the processing of information by the nervous system.
\end{abstract}

Key words: Caudal photoreceptor - Crustaceans - Cherax quadricarinatus - Spontaneous activity - Photoresponse

\begin{abstract}
Abbreviations: CPR, caudal photoreceptor; CPR-L, caudal photoreceptor-left; CPR-R, caudal photoreceptor-right; $\triangle \mathrm{F}$, variation in frequency of action potentials per second for CPR activity; LWS, long-wavelength-sensitive opsin (530 nm); SWS, short-wavelength-sensitive opsin (440 nm); $\mathrm{AG}$, abdominal ganglion.
\end{abstract}

\section{Introduction}

In the freshwater crayfish, light preferences for locomotive behavior are mediated by the compound eyes, brain photoreceptors, and caudal photoreceptors (CPRs). This photosensitive system modulates locomotive activity in the animal by different physiological mechanisms (Fernández de Miguel

Correspondence to: Leonardo Rodríguez-Sosa, Departamento de Fisiología, Facultad de Medicina, UNAM, Av. Universidad 3000, C. P. 04510. Ciudad de México, México

E-mail: lrsosa@unam.mx and Aréchiga 1992; Aréchiga and Rodríguez-Sosa 1997; Kozák et al. 2009; Sullivan et al. 2009; Strauss and Dircksen 2010; Rodríguez-Sosa et al. 2017). Variations in light intensity throughout the day may expand by approximately nine logarithmic intensity units (Nilsson 2009).

The current study focuses on the caudal photoreceptors. The CPRs consist of a pair of bilateral photosensitive neurons among the 600 to 700 neurons that comprise the entire $6^{\text {th }}$ abdominal ganglion $\left(6^{\text {th }} \mathrm{AG}\right)$. The caudal photoreceptor produces small contralateral dendritic branches and the axons send projections along the ganglia chain, passing through the thoracic, subesophageal, and reaching the supraesophageal 
ganglia. The CPR is in the anterior part of the ventral side of the ganglion and in the five to six abdominal connectives assumes a ventral position of the cord (Wilkens and Larimer 1972, 1976; Simon and Edwards 1990). The CPRs display a spontaneous action potential for darkness, making a phasic-tonic activity in direct response to high levels of illumination. The molecular expression of the CPR photopigments has been suggested (Kingston and Cronin 2015); its subcellular localization (Uchizono 1962), as well as the phototransduction mechanism (Kruszewska and Larimer 1993), are only partly known. Intracellular recordings from CPR dendrites suggest that they are the sites of light transduction (Wilkens 1988). Additionally, these neurons respond trans-synaptically to mechanosensory inputs (Wilkens and Larimer 1972; 1976; Wilkens 1988; Bahar and Moss 2004). The CPR has been studied in different decapod crustaceans from marine and freshwater sites, revealing that photosensitive neurons also show similarities in their anatomic location and light responsiveness (Wilkens and Larimer 1976).

Recently, the spontaneous activity and photoresponse of CPRs has been studied in crayfish (Rodríguez-Sosa et al. 2008; Villagran-Vargas et al. 2013; Nesbit et al. 2015). When tested for light pulses of different intensities, the CPR responsiveness at night is almost one log unit greater than in daytime (see Rodríguez-Sosa et al. 2008). Furthermore, the CPR also behaves as a circadian pacemaker, which is involved in the synchronization of the diurnal rhythm of locomotive activity in the crayfish (see Rodríguez-Sosa et al. 2008, 2012; Strauss and Dircksen 2010). However, in these studies, no information is available for identifying the lateral location of the CPR.

On the other hand, different studies have shown sensory and motor asymmetries in behavior, as well as asymmetries in the nervous system, in invertebrates (see Frasnelli et al. 2012, 2013). In crustacean decapods, left and right asymmetries have been reported in conspicuous anatomical regions, such as the cephalothorax (Tobo et al. 2012), claws (Palmer 2016), and the tail curvature (Takeuchi et al. 2008). Some functional properties showed asymmetry. Takeuchi et al. (2008) suggested that shrimp has an innate left-right lateral movement ability in terms of their escape direction, which may affect prey-predator interactions. Crayfish use their limbs during aggression; asymmetries of limb performance could affect this behavior (Angilletta and Wilson 2012).

Levin et al. (2016) have suggested that left and right asymmetries in organisms arise from embryogenesis. $6^{\text {th }} \mathrm{AG}$ develops from two embryonic neuromeres in the crayfish Procambarus clarkii (Kondoh and Hisada 1986; Mulloney et al. 2003), and the CPR-axons are in the ventral cord, in Wiersma area 82 for the left hemicord, and in area 79 of the right hemicord of the cord map of the crayfish (Hermann 1972). Other reports showed electrophysiological record- ings from the two caudal photoreceptors (Kennedy 1963; Hermann and Olsen 1968). For each CPR, micro-regional illumination on the ventral surface of the $6^{\text {th }}$ AG estimated that the light sensitive area was 2-3 times greater on the left side than the right side of the crayfish Orconectes virilis (Hermann and Olsen 1968).

Although the physiology of these photosensitive neurons is well established, their comparative properties between the left and right caudal photoreceptor (CPR-L and CPR-R respectively) has not been thoroughly studied. Thus, we simultaneously explored the spontaneous discharge of action potentials in darkness and during photoresponse from the CPR-L and the CPR-R, and the effect of temperature in both activities of the left and right CPRs. This study was performed using an extracellular recording procedure in the isolated $6^{\text {th }} \mathrm{AG}$ of the crayfish Cherax quadricarinatus.

\section{Materials and Methods}

\section{Experimental animals}

We used ten adult crayfish Cherax quadricarinatus with weights ranging from 30 to $40 \mathrm{~g}$, and carapace lengths ranging from 11 to $13 \mathrm{~cm}$ from the rostrum to telson. Animals were acquired at a local provider and were kept in the laboratory in aerated water containers for two weeks before the experiments, with a program of 12:12-hours of light-dark cycles and the light intensity during the daytime period had 560 Lux. The animals had free access to vegetables and dried fish as food. Animal care was in accordance with the policies of the Society for Neuroscience (2017), and the protocol was approved by the ethical committee, Faculty of Medicine, National Autonomous University of Mexico, Mexico.

\section{Extracellular recordings}

Electrophysiological experiments were performed in accordance with a previously described procedure (Rodríguez-Sosa et al. 2006, 2011). Briefly, the AG chain was removed from the crayfish under the ice-cold modified physiological saline Van Harreveld (VH) solution (Van Harreveld 1936), (205 $\mathrm{mM} \mathrm{NaCl}, 5.4 \mathrm{mM} \mathrm{KCl}, 2.6 \mathrm{mM} \mathrm{MgCl}$, $13.5 \mathrm{mM} \mathrm{CaCl}_{2}$, and $10 \mathrm{mM} \mathrm{HEPES}$ ), and $\mathrm{pH}$ was adjusted to 7.4. The isolated $6^{\text {th }} \mathrm{AG}$ and some nerve bundles between the $5^{\text {th }}$ and the $6^{\text {th }}$ AG were dissected using a stereoscopic microscope. Then, the biological preparation was transferred to the recording chamber mounted on a microscope (SMZ800, Nikon) with VH saline solution perfusion (approximately $1 \mathrm{ml} / \mathrm{min}$ ) in the dark. Usually, the $6^{\text {th }}$ AG was maintained at room temperature $\left(24 \pm 1.7^{\circ} \mathrm{C}\right)$. For some experiments, these ganglia were stabilized at $25^{\circ} \mathrm{C}$ or $15^{\circ} \mathrm{C}$ by supplying the water from a temperature-regulated bath. 
As shown in Figure 1A, B, extracellular recordings were made with suction electrodes filled with the saline solution of $\mathrm{VH}$, and these electrodes were positioned on the right and left hemicord, respectively, and the reference electrode (Ag-AgCl) was in the bath solution. Signal recordings were made by using AC amplifiers (EX1, Dagan), filtered at 30 $\mathrm{Hz}$ to $10 \mathrm{kHz}$ with a band-pass filter system, and displayed on an oscilloscope (Gould 1604). As described earlier, the photoresponse of the CPR depends on light intensity (15-900 Lux) and the time of day (see Rodríguez-Sosa et al. 2008). The current study was made between 11:00 to 14:00 hours, using a constant white light pulse (700 Lux, $4 \mathrm{~s})$. It allowed us to compare easily the light-induced activity in the left-right CRPs in the isolated ganglion of different crayfish. Light pulses were delivered by a photostimulator (Grass PS33), which produced white incandescent light. The calibration of intensity was performed with a photographic light meter (Goossen, model Luna-Pro, Germany). Electrophysiological data were recorded onto a computer using Spike2 software and a Micro-1401 AD board (CED, Cambridge, UK). The sorting spike from the CPRs was made off-line by the Spike2 software.

\section{Statistical analyses}

The spontaneous activity of the CPRs in darkness was measured by the frequency of action potentials per second and described with median values and interquartile range (IQR). The light photoresponse was normalized as follows: we calculated the variation in the frequency of action potentials per second for the CPR activity, and it was subtracted from the mean value of its basal activity of the light-induced activity and normalized the value maximum of one caudal photoreceptor in each $6^{\text {th }}$ AG. The findings were expressed as $\Delta \mathrm{F}(\%)$, and median values and their IQR were calculated.

The firing rates from the CPRs were analyzed with the normality test in accordance with the Shapiro-Wilk test or Kolmogorov-Smirnov. The firing rate from the CPRs did not have a normal distribution; Friedman's test was used. Additionally, the firing rate of the spontaneous activity (impulses/s), and the photoresponse $\Delta \mathrm{F}(\%)$, between left and right CPR activities for two-samples were also compared using the Mann-Whitney test. All statistical analyses were established with $p<0.05$. Statistical analyses were done with Origin (OriginLab, Northampton, MA).
A

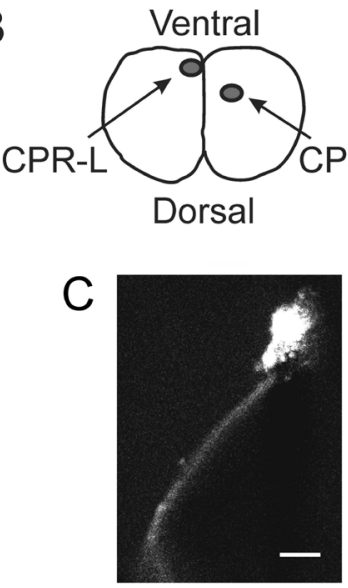

D
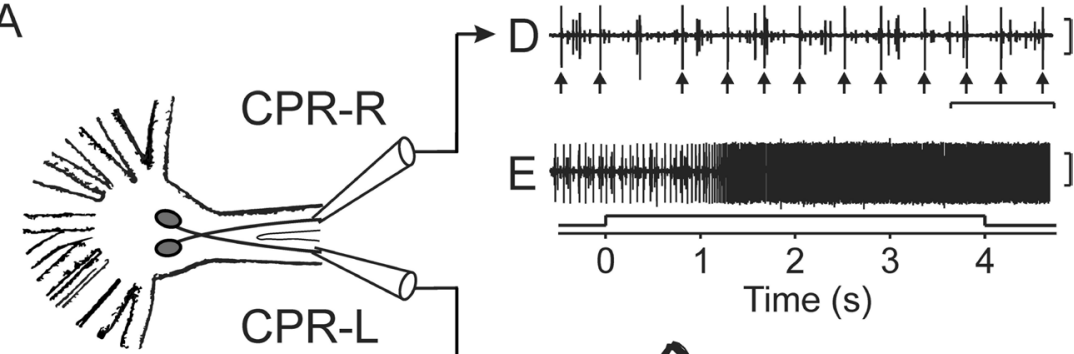

E

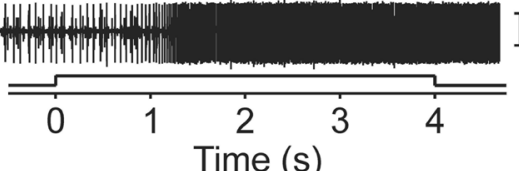

$\mathrm{F}$

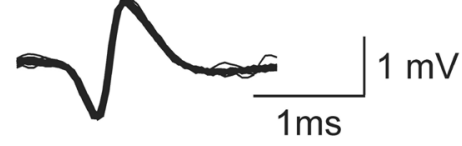
PR-R
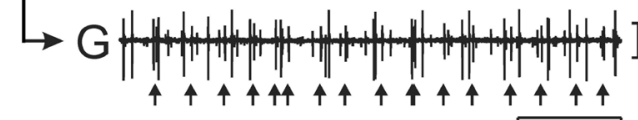

$\mathrm{H}$

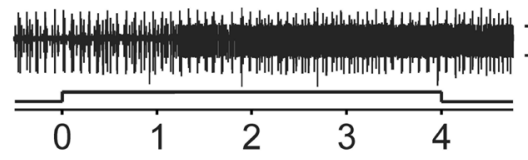

Time (s)
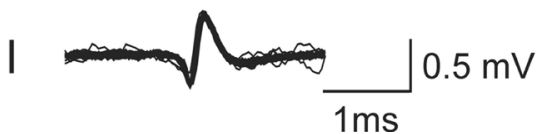

Figure 1. Electrophysiological recordings of the left and right caudal photoreceptor (CPR-L and CPR-R respectively) from the isolated ganglion. A. Scheme of the $6^{\text {th }}$ AG showing how the cord between the sixth and fifth abdominal ganglia was microdissected into left and right hemicords. Extracellular recordings were made with a suction electrode. B. Approximate location of the CPR axons in the connective tract between the sixth and fifth abdominal ganglia (modified from Hermann 1972). C. Microphotography showing a putative CPR after being backfilled with Lucifer yellow (modified from Rodríguez-Sosa et al. 2006). D. Recordings of multi-unit activity from the right hemicord. Arrows show basal activity of CPR-R. Scale bars: $0.2 \mathrm{~s}$ and $1 \mathrm{mV}$ as an insert. E. Light-induced activity from CPR-R. Note the time course of the variation in the frequency of action potentials induced by a pulse of white light. The scale vertical of $1 \mathrm{mV}$ as an insert. At bottom, a light pulse is displayed. F. Overdraw waveform of 30 spikes from the CPR-R. G. Recordings of multi-unit activity from the hemicord-left.

The spontaneous activity from CPR-L is indicated by the arrows. Scale bars: $0.2 \mathrm{~s}$ and $0.5 \mathrm{mV}$ are indicated. H. Light-induced activity from CPR-L. The scale vertical at $0.5 \mathrm{mV}$ is indicated. At the bottom, a light pulse is displayed (700 Lux, $4 \mathrm{~s}$ ). I. Overdraw waveform of 30 spikes from the CPR-L. 


\section{Results}

Analysis of CPR activities from multiunit activity in the isolated sixth abdominal ganglion

The first step in this study consisted of making simultaneous recordings and identifying the CPR-L and CPR-R activities in the isolated sixth abdominal ganglion (Fig. 1). First, extracellular recordings were made with a suction electrode placed in the left-right hemicord (see Figs. 1A, B). Second, multiunit activity from the continuous electrophysiological recordings (see Figs. 1D, E, G, H) was examined onto the spike classification algorithm; differences in waveform, amplitude, and duration formed part of a basis for separating spikes from different putative neurons. Usually, the spikes from the left and right CPRs had extracellular potentials biphasic, negative-positive shapes and different amplitudes (see Figs. 1F, I). Third, the analysis of the neuronal population allows this characterization of multi-spike trains, and it was carried out in accordance with their temporal firing patterns of the left and right CPRs in response to the light stimulus (see Figs. 1E, H).

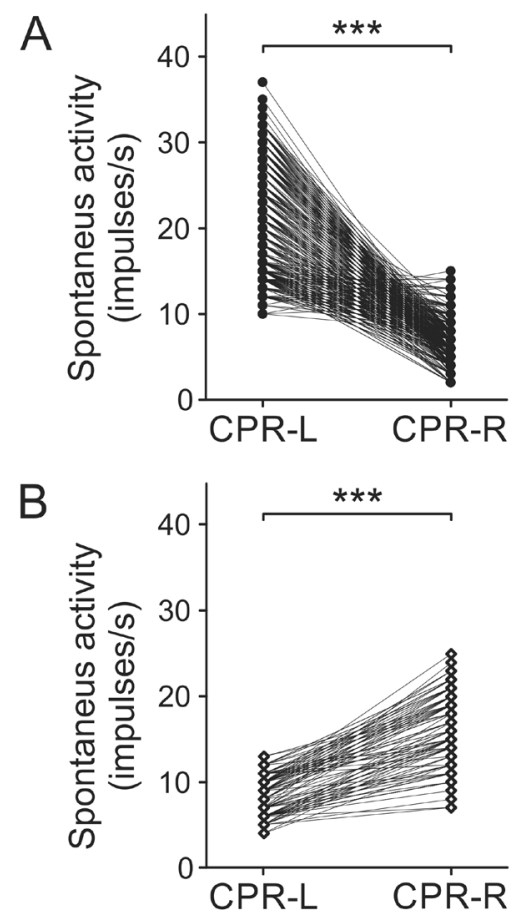

Figure 2. The left and right CPR activity in darkness plotted as the line series (mean values). Note the distinct activity between the CPR-L and the CPR-R in two groups of ganglia. A. CPR-L has a higher activity regarding the CPR-R. B. Another group where CPR-R expresses a higher spontaneous activity with respect to the CPR-L. ${ }^{* *} p<0.001$, differences between groups were significant.
Asymmetry from left and right CPR firing rate in darkness

The left and right CPR activities in darkness at room temperature were recorded in a group of nine isolated ganglia. CPRs showed a tonic firing rate, and this activity had an asymmetry between CPR-L and CPR-R. Data analysis was carried out in bins of one second and computed in one minute, and showed two subgroups of CPRs. We observed one subgroup (70\%) in which CPR-L had a spontaneous firing rate median of $18($ IRQ $=11)$ impulses $/ \mathrm{s}$ and CPR-R had a median of $6($ IRQ $=3)$ impulses/s. The pairwise comparison of the Mann-Whitney $\mathrm{U}$ test was significant; $\mathrm{Z}=-24.930, p<0.001$ (see Fig. 2A). In another subgroup (20\%), CPR-R had a spontaneous firing rate median of 15 (IRQ = 7) impulses/s, and CPR-L had a median of 8 $(I R Q=4) \mathrm{impulses} / \mathrm{s}$. The pairwise comparison of the Mann-Whitney $U$ test was significant; $Z=-11.966, p<$ 0.001 (see Fig. 2B).

\section{Asymmetric photoresponse in the left and right CPR to white light pulses}

The light and induced activity was studied in a group of seven biological preparations. The time course of the variation in the frequency of the action potentials $(\Delta \mathrm{F}(\%))$ induced by a pulse of white light (700 Lux, 4 s) showed a latency of $\sim 1.2-1.5 \mathrm{~s}$. The time to produce the maximum photoresponse after applying the luminous stimulus was 2.4-3.2 s. Data analysis of the light-induced activities in the left and right CPRs from 10 to 30 seconds showed two subgroups of ganglia.

In the first group, the differences between left and right CPRs before light-pulses were not found $(Z=-0.932, p>$ $0.05)$. The CPR activities showed differences in time intervals after light-pulses: $\mathrm{X}^{2}(2)=69.644, p<0.001$ to CPR-L, and $\mathrm{X}^{2}(2)=64.490, p<0.001$ to CPR-R. Then, statistically significant differences between CPR-L and CPR-R were noted between 10 to $30 \mathrm{~s}$. The pairwise comparison of the Mann-Whitney $U$ test was significant. In the interval from 0 to $10 \mathrm{~s}$, there was a significant statically difference $Z=-3.090$, $p<0.01$, from 10 to $20 \mathrm{~s}$ the difference was $Z=-2.736, p<$ 0.01 ; and from 20 to $30 \mathrm{~s}$ the difference was $Z=-2.663, p<$ 0.01 (see Fig. 3A).

In the other group, the differences between left and right CPRs before light-pulses were not observed $(Z=-0.509, p>$ $0.05)$. The CPR activities showed differences in time intervals after light-pulses: $\mathrm{X}^{2}(3)=115.194, p<0.001$ to CPR-L, and $\mathrm{X}^{2}(3)=142.87, p<0.001$ to CPR-R. However, for this group, statistically significant differences between CPR-L and CPR-R were noted only at $10 \mathrm{~s}$. The pairwise comparison of the Mann-Whitney $U$ test was significant; $Z=-2.684, p<$ 0.01 (see Fig. 3B). 
Differential effects of temperature on the left and right $C P R$ activities

The next step in this study consisted of testing the effect of temperature on spontaneous activity in the darkness for each $\mathrm{CPR}$. The experiment was started at $25^{\circ} \mathrm{C}$ and then lowered to $15^{\circ} \mathrm{C}$, from a group of two biological preparations. Figure $4 \mathrm{~A}$ shows the time course of the spontaneous activity in darkness at $25^{\circ} \mathrm{C}$ in both CPRs. CPR-L had a spontaneous firing rate median of 33 (IRQ $=14)$ impulses/s $(n=3)$, and CPR-R had a median of $7(I R Q=3)$ impulses/s. The pairwise comparison of the Mann-Whitney $\mathrm{U}$ test was significant; $\mathrm{Z}=$ $-16.433, p<0.001$. Figure 4B illustrates the time course of the spontaneous activity in darkness at $15^{\circ} \mathrm{C}$ for the CPRs. CPR-L had a spontaneous firing rate median of $14(\mathrm{IRQ}=11)$ impulses/s. CPR-R had a median of $5(\mathrm{IRQ}=3) \mathrm{impulses} / \mathrm{s}$.
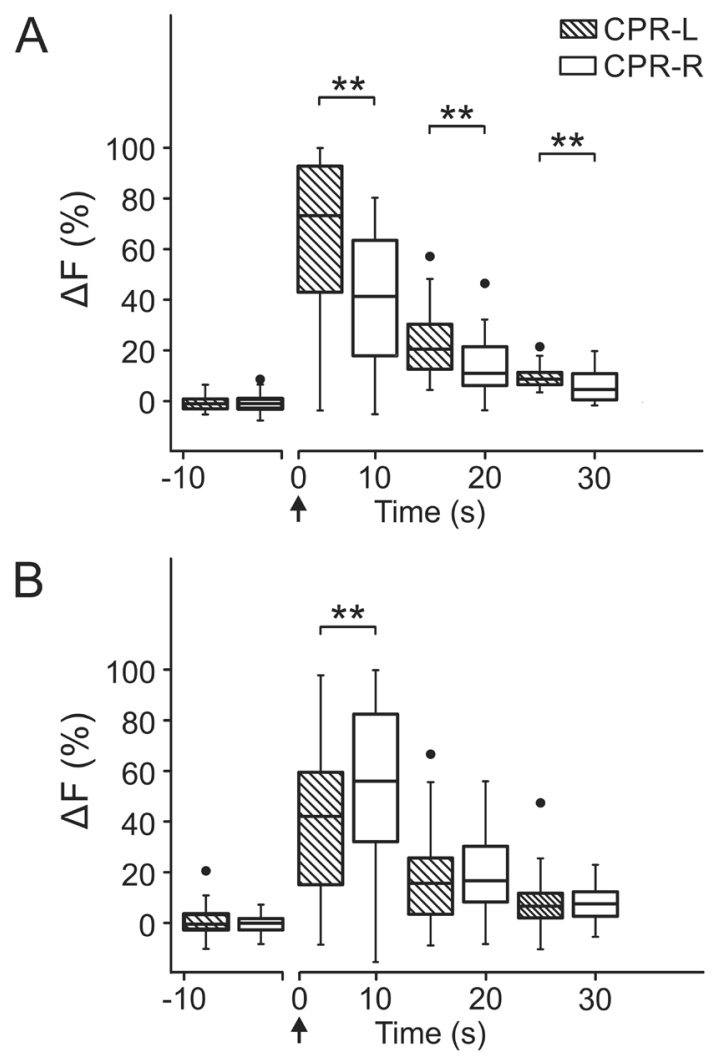

Figure 3. Time course of the light-induced activity from the CPR. A. The box plot for CPR-L (dashed box) compared to CPR-R (clear box). At the bottom, the pulse of white light is indicated by the arrow. Ordinates, $\Delta \mathrm{F}(\%)$, box values represent the median and interquartile range. Note the major photoresponse from CPR-L compared to CPR-R. B. Light-induced activity in another group of CPR, a major increased photoresponse from the CPR-R versus to the CPR-L. ${ }^{* *} p<0.01$, difference between groups was significant.
The pairwise comparison of the Mann-Whitney $U$ test was significant; $Z=-14.552, p<0.001$.

The light-induced activity in both $\mathrm{CPRs}$ at $25^{\circ} \mathrm{C}$ showed a latency of $\sim 0.7-1.5 \mathrm{~s}$. The time to produce the maximum photoresponse after applying the luminous stimulus was 3.5-4.0 s.

Then, this activity had a gradual recovery. The time course of light-induced activity from CPRs at $25^{\circ} \mathrm{C}$ is shown in Figure 5A. No differences were found between left and right CPRs before light-pulses $(Z=-0.533, p>0.05)$. CPR activities showed differences in time intervals after light-pulses; $\mathrm{X}^{2}(2)=65.909, p<0.001$ to CPR-L, and $\mathrm{X}^{2}(2)=71.465, p<$ 0.001 to CPR-R. However, for this group, statistically significant differences between CPR-L and CPR-R were observed only at $10 \mathrm{~s}$. The pairwise comparison of the Mann-Whitney $\mathrm{U}$ test was significant; $\mathrm{Z}=-2.070, p<0.05$ (Fig. 5A).
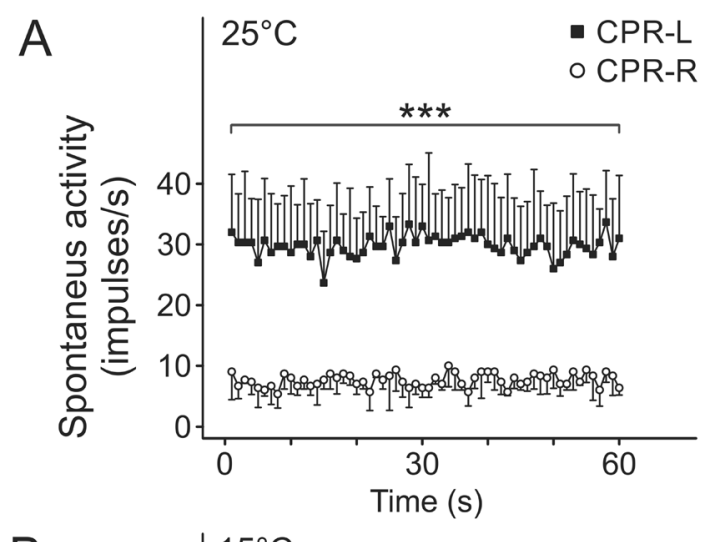

B

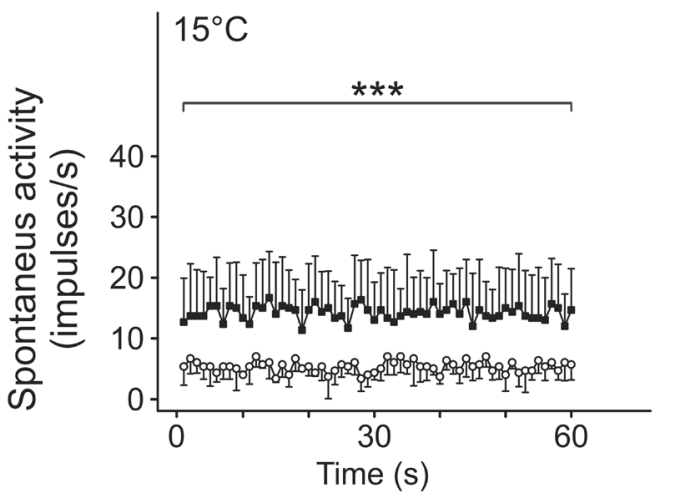

Figure 4. Effect of temperature on spontaneous activity in the darkness from CPR. Time course from left and right CPR for the spontaneous firing rate in the dark at $25^{\circ} \mathrm{C}$ and $15^{\circ} \mathrm{C}$. Caudal photoreceptor on the left side (in filled rectangles) and caudal photoreceptor on the right side (in clear circles). Ordinates, firing rate (impulses per second), values represent mean \pm standard deviation. A. Note the high activity from CPR-L at $25^{\circ} \mathrm{C}$. B. The firing rate of the left and right CPRs at $15^{\circ} \mathrm{C}$. Both CPRs showed lower spontaneous activity under this condition. ${ }^{* *} p<0.001$, the difference between groups was found to be significant. 


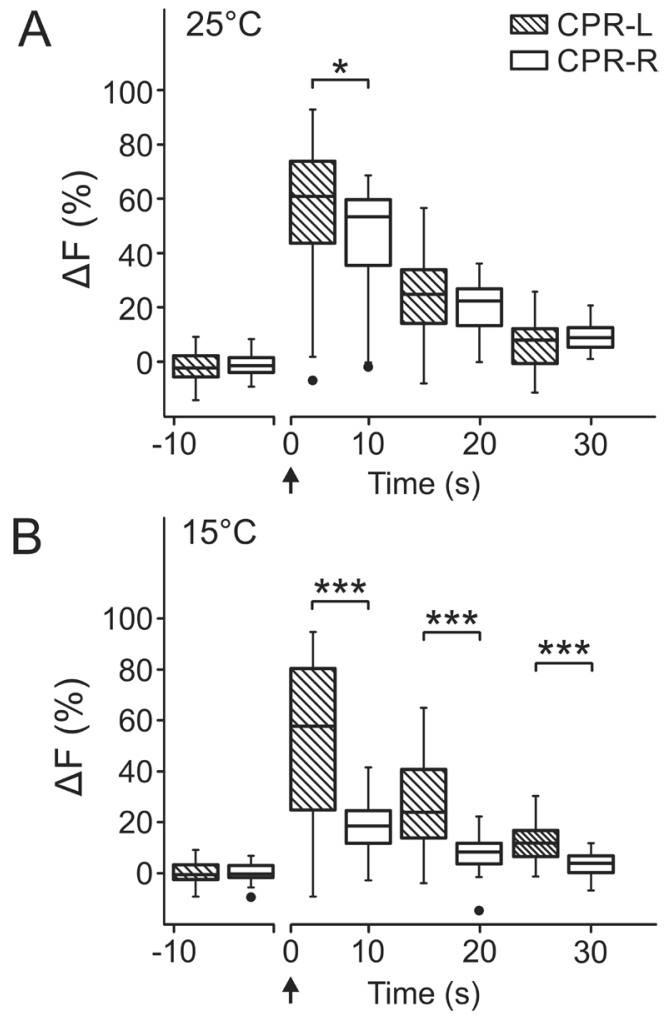

Figure 5. Effect of temperature on the light-induced activity of the CPR. A. Box plot for CPR-L (dashed box) compared to CPR$\mathrm{R}$ (clear box) at $25^{\circ} \mathrm{C}$. At the bottom, the pulse of white light is indicated by the arrow (700 Lux, $4 \mathrm{~s}$ ). Ordinates, $\Delta \mathrm{F}(\%)$, box values represent the median and interquartile range. Note the major photoresponse from CPR-L compared to CPR-R. B. The light-induced activity of the CPRs at $15^{\circ} \mathrm{C}$ showed lower activities. Note the major photoresponse from CPR-L compared to CPR-R. ${ }^{*} p<0.05 ;{ }^{* *} p<0.001$, differences between groups were found to be significant.

The photoresponse was recorded by lowering the temperature in these same ganglia to $15^{\circ} \mathrm{C}$. As seen in Figure $5 \mathrm{~B}$, no differences between left and right CPRs before lightpulses were found $(Z=-0.296, p>0.05)$. In both CPRs, the photoresponse showed similar values for latency, the time to produce the maximum activity after the light stimulus above described. The CPR activities showed differences in time intervals after light-pulses: $\mathrm{X}^{2}(2)=52.691, p<0.001$ to CPR-L, and $\mathrm{X}^{2}(2)=45.157, p<0.001$ to CPR-R. Then, statistically significant differences between CPR-L and CPR-R were noted from 10 to $30 \mathrm{~s}$. The pairwise comparison of the Mann-Whitney U test was significant. In the interval from 0 to $10 \mathrm{~s}$, there was a significant statically difference $Z=-3.667$, $p<0.001$; from 10 to $20 \mathrm{~s}$ the difference was $Z=-4.289, p<$ 0.001 ; and from 20 to $30 \mathrm{~s}$ the difference was $Z=-4.378, p<$ 0.001 (see Fig. 5B).

\section{Discussion}

The current study focused on comparing spike trains simultaneously measured in the left and right CPRs in the isolated sixth abdominal ganglion of the crayfish Cherax quadricarinatus. The CPR-L and CPR-R showed asymmetrical firing rates for the spontaneous activity in darkness and in the light-induced activities. Moreover, the temperature had a differential influence on CPR activities.

The electrophysiological procedure was based on the population analysis of neuronal recordings (see Quian Quiroga and Panzeri 2009), and provides a view on how spike activities from the left and right CRPs were evaluated. Typically, the left and right CPR extracellular action potentials had a biphasic, negative-positive shape (Figs. 1F, I), which is in accordance with the volume conductor theory (see Heinricher 2004), suggesting that the source of the currents of the spikes were generated near the initial segment of the axon-soma of the CPR, then being propagated into the axon of left and right CPRs, with a conduction velocity of $4.25 \pm$ $0.2 \mathrm{~m} / \mathrm{s}$ (Villagran-Vargas et al. 2013). Thus, these waveforms suggest that electrodes were located near the source of the currents for the spikes.

As mentioned earlier, the asymmetry in the properties of the anatomy, nervous system and behavior are the themes of study in crustaceans (Takeuchi et al. 2008; Angilletta and Wilson 2012; Palmer 2016). Other authors have also reported some differences in the recordings from the two CPRs in darkness with respect to their light-induced activity (Kennedy 1963; Hermann and Olsen 1968). We emphasized here laterality, and compared the spontaneous firing rate in darkness and the photoresponses from CPR-L and CPR-R. In the dark, we observed two subgroups of ganglia, where the main subgroup (70\%) showed that CPR-L had a higher spontaneous firing rate compared with CPR-R (Fig. 2). In other studies, it was reported that the mechanosensory system has a role in excitatory and inhibitory effects in CPRs which is mediated trans-synaptically (Kennedy 1963; Hermann and Olsen 1968; Wilkens and Larimer 1976; Pei et al. 1996). The CPR responded preferentially to water-jet stimuli presented ipsilaterally to the CPR (Tautz and Plummer 1994). Additionally, this photosensitive neuron has an electrical activity that is like an endogenous pacemaker (Larimer 2000), and behaves as an oscillator on the circadian system of the crayfish (Rodríguez-Sosa 2008, 2012; Strauss and Dircksen 2010). In addition to this, we noted the presence of two subgroups of $6^{\text {th }}$ AG with asymmetric light-induced activity. This can be seen in Figure 3, where, in 70\% of the ganglia, the CPR-R showed the maximum photoresponse. In the other $30 \%$ of ganglia, the CPR-L had the greatest light-induced activity. Kingston and Cronin (2015) described the expression of two retinal photopigments in the crayfish $P$. clarkii. These opsins have maximum sensitivity, $440 \mathrm{~nm}$ short wavelength (SWS) 
and $530 \mathrm{~nm}$ long wavelength (LWS), suggesting that these two opsin proteins may participate in caudal photoreception in the $6^{\text {th }}$ AG. We may consider a dynamic balance in the content of the opsins in the two groups of CPRs. Battelle (2016) has reviewed the physiology of opsins with apparently low expression levels in another extraocular photoreceptor model. Moreover, light enhances the transduction of hydrodynamic stimuli, possibly through fluctuations in membrane potential of the CPR (Pei et al. 1996; Bahar and Moss 2003). Furthermore, some substances have been postulated as a neurotransmitter or neuromodulators on CPR activities. 5-hydroxytryptamine (Rodríguez-Sosa 2006, 2007, 2008), dopamine (Rodríguez-Sosa et al. 2011) and acetylcholine (Hermann and Skiles 1969) showed different effects on the firing rate in darkness and in the light-induced activity of the CPRs. It is interesting that the asymmetric neurotransmitter release has been reported in olfactory receptor neurons in Drosophila (Gaudry et al. 2013).

Recently, preference and tolerance to temperature in crayfish has been studied (García-Guerrero et al. 2013; Westhoff and Rosenberger 2016). The temperature dependence for the CPR activities has been described (Larimer 1967; Belanger 1988; Rodríguez-Sosa et al. 2008), but the activity of CPR-L and CPR-R has not been specified. The current results showed a differential effect of temperature in CPR-L and CPR-R in darkness and in light-induced activity (Figs. 4, 5, respectively). This assay was performed (Figs. 4,5 ) set with a temperature range from $15^{\circ} \mathrm{C}$ to $25^{\circ} \mathrm{C}$, which are between the temperature ranges of $12^{\circ} \mathrm{C}$ to $33^{\circ} \mathrm{C}$ found in the native environment of the crayfish C. quadricarinatus (Masser and Rouse 1997). Interestingly, another author has reported temperature robustness in neuronal oscillators (Caplan et al. 2014). The transient receptor potential (TRP) channels have been postulated to be involved in thermal sensitivity in crayfish P. clarkii (Ríos-Cano et al. 2014), but the mechanism for temperature sensitivity in CPRs is not known.

Overall, the results in the present study provide insight into the asymmetry of CPR-L and CPR-R in both activities. These results suggest a starting point for future research into the functional asymmetry of the CPRs in circadian rhythms and locomotive behavior in crayfish. This includes possible mechanisms involved in asymmetrical activities from the CPRs, as the potential of membrane fluctuations, the expression of opsins (LWS, SWS), and the TRP channels. Other studies should also focus on characterizing the physiological role of this asymmetry on left and right CPR activities in the crayfish.

\section{Conclusions}

The procedure described here for the analysis of neuronal recordings was reproducible for characterizing left and right CPR activities in the isolated ganglion. The results here provide insight into the asymmetry and spontaneous firing rate in darkness and in light-induced activity in CPRs. CPR-L and CPR-R showed differential thermal-sensitivity. These asymmetries may be considered a new functional property of CPRs.

Acknowledgments. This study was supported by Facultad de Medicina-UNAM grant 086/2016, and PAPIIT-UNAM Grant IN214817 to LRS. The authors wish to acknowledge Mr. Víctor Anaya for their invaluable technical help.

Conflict of interest. The authors declare no conflict of interest.

\section{References}

Angilletta JM, Wilson RS (2012): Cryptic asymmetry: unreliable signals mask asymmetric performance of crayfish weapons. Biol. Lett. 8, 551-553 https://doi.org/10.1098/rsbl.2012.0029

Aréchiga H, Rodríguez-Sosa L (1997): Coupling of environmental and endogenous factors in the control of rhythmic behaviour in decapod crustaceans. J. Mar. Biolog. Assoc. U.K. 77, 17-29 https://doi.org/10.1017/S0025315400033750

Bahar S, Moss F (2003): Stochastic phase synchronization in the crayfish mechanoreceptor/photoreceptor system. Chaos 131, 138-144 https://doi.org/10.1063/1.1501899

Bahar S, Moss F (2004): Stochastic resonance and synchronization in the crayfish caudal photoreceptor. Math. Biosci. 188, 81-97 https://doi.org/10.1016/j.mbs.2003.09.004

Battelle BA (2016): Simple eyes, extraocular photoreceptors and opsins in the American horseshoe crab. Integr. Comp. Biol. 56, 809-819 https://doi.org/10.1093/icb/icw093

Belanger JH (1988): Temperature acclimation of the caudal photoreceptor response in the crayfish Orconectes rusticus (Girard). Can. J. Zool. 66, 1168-1171 https://doi.org/10.1139/z88-170

Caplan JS, Williams AH, Marder E (2014): Many parameter sets in a multicompartment model oscillator are robust to temperature perturbations. J. Neurosci. 34, 4963-4975 https://doi.org/10.1523/JNEUROSCI.0280-14.2014

Fernández de Miguel F, Aréchiga H (1992): Sensory inputs mediating two opposite behavioural responses to light in the crayfish Procambarus clarkii. J. Exp. Biol. 164, 153-169

Frasnelli E (2013): Brain and behavioral lateralization in invertebrates. Front Psychol. 4, 939 https://doi.org/10.3389/fpsyg.2013.00939

Frasnelli E, Vallortigara G, Rogers LJ (2012): Left-right asymmetries of behaviour and nervous system in invertebrates. Neurosci. Biobehav. Rev. 36, 1273-1291 https://doi.org/10.1016/j.neubiorev.2012.02.006

García-Guerrero M, Hernández-Sandoval P, Ordu-a-Rojas J, Cortés-Jacinto E (2013): Effect of temperature on weight increase, survival, and thermal preference of juvenile red claw crayfish Cherax quadricarinatus. Hidrobiologica 23, 73-81 
Gaudry Q, Hong EJ, Kain J, de Bivort BL, Wilson RI (2013): Asymmetric neurotransmitter release enables rapid odour lateralization in Drosophila. Nature 493, 424-428 https://doi.org/10.1038/nature11747

Heinricher M (2004): Principles of extracellular single-unit recording. In: Microelectrode Recording in Movement Disorder Surgery. (Ed. Izrael Z. and Burchiel K. J.), New York

Hermann HT, Olsen RE (1968): Afferent stochastic modulation of crayfish caudal photoreceptor units. J. Gen. Physiol. 51, 534-551 https://doi.org/10.1085/jgp.51.4.534

Hermann HT, Skiles MS (1969): Cholinergic inhibition of the crayfish caudal photoreceptor. Comp. Biochem. Physiol. 31, $575-588$ https://doi.org/10.1016/0010-406X(69)90059-0

Hermann HT (1972): Analysis of the properties of the crayfish caudal photoreceptor (PRU-photoreceptor unit). In: Experiments in Physiology and Biochemistry. (Ed. Kerkut G.A.), Vol. 3, pp. 155-192, Academic Press, London

Kruszewska B, Larimer JL (1993): Specific second messengers activate the caudal photoreceptor of crayfish. Brain Res. 618, 32-40 https://doi.org/10.1016/0006-8993(93)90425-M

Kennedy D (1963): Physiology of photoreceptor neurons in the abdominal nerve cord of the crayfish. J. Gen. Physiol. 46, 551-572 https://doi.org/10.1085/jgp.46.3.551

Kingston ACN, Cronin TW (2015): Short- and long-wavelengthsensitive opsins are involved in photoreception both in the retina and throughout the central nervous system of crayfish. J. Comp. Physiol. A 201, 1137-1145 https://doi.org/10.1007/s00359-015-1043-2

Kondoh Y, Hisada M (1986): Neuroanatomy of the terminal (sixth abdominal) ganglion of the crayfish, Procambarus clarkii (Girard). Cell Tissue Res. 243, 273-288 https://doi.org/10.1007/BF00251041

Kozák P, Martín Gallardo J, Escudero García JC (2009): Light preferences of red swamp crayfish (Procambarus clarkii). Hydrobiologia 636, 499-503

https://doi.org/10.1007/s10750-009-9937-z

Larimer JL (1967): The effects of temperature on the activity of the caudal photoreceptor. Comp. Biochem. Physiol. 22, 683-700 h https://doi.org/10.1016/0010-406X(67)90762-1

Larimer JL (2000): The interneurons of the abdominal positioning system of the crayfish. Brain Evol. 55, 241-247 https://doi.org/10.1159/000006658

Levin M, Klar AJS, Ramsdell AF (2016): Introduction to provocative questions in left-right asymmetry. Philos. Trans. R. Soc. B 371, 20150399 https://doi.org/10.1098/rstb.2015.0399

Masser M, Rouse D (1997): Australian red claw crayfish. Southern Regional Aquaculture Center of the United States, Publication No. 244, 8 p, Auburn, AL

Mulloney B, Tschuluun N, Hall WM (2003): Architectonics of crayfish ganglia. Microsc. Res. Tech. 60, 253-265 https://doi.org/10.1002/jemt.10265

Nesbit SC, Van Hoof AG, Le CC, Dearworth JR (2015): Extracellular recording of light responses from optic nerve fibers and the caudal photoreceptor in the crayfish. J. Undergrad. Neurosci. Educ. 14, A29-38
Nilsson DE (2009): The evolution of eyes and visually guided behaviour. Philos. Trans. R. Soc. B. 364, 2833-2847 https://doi.org/10.1098/rstb.2009.0083

Pei X, Wilkens LA, Moss F (1996): Light enhances hydrodynamic signaling in the ultimodal caudal photoreceptor interneurons of the crayfish. J. Neurophysiol. 76, 3002-3011

Palmer AR (2016): What determines direction of asymmetry: genes, environment or chance? Philos. Trans. R. Soc. B 371, 20150417 https://doi.org/10.1098/rstb.2015.0417

Quian Quiroga R, Panzeri S (2009): Extracting information from neuronal populations: information theory and decoding approaches. Nat. Rev. Neurosci. 10, 173-185 https://doi.org/10.1038/nrn2578

Ríos-Cano S, García U, Rodríguez-Sosa L, Anaya V, CalderónRosete G (2014): Thermal response of a motoneuron cluster: possible participation of TRP channels and circadian changes in spontaneous activity under constant conditions. In: Horizon in Neuroscience Research. (Eds. Costa A. and Villalba E.), pp. 115-126, Nova Science Publishers, Inc, N. Y.

Rodríguez-Sosa L, Calderón-Rosete G, Villalobos MGP, Zamora EM, González VA (2006): Serotonin modulation of caudal photoreceptor in crayfish. Comp. Biochem. Physiol. C 142, 220-230 https://doi.org/10.1016/j.cbpc.2005.10.006

Rodríguez-Sosa L, Calderón-Rosete G, Flores G, Porras MG (2007): Serotonin-caused phase shift of circadian rhythmicity in a photosensitive neuron. Synapse 61, 801-808 https://doi.org/10.1002/syn.20425

Rodríguez-Sosa L, Calderón-Rosete G, y Flores G (2008): Circadian and ultradian rhythms in the crayfish caudal photoreceptor. Synapse 62, 643-652 https://doi.org/10.1002/syn.20540

Rodríguez-Sosa L, Calderón-Rosete G, Calvillo ME, Guevara J, Flores G (2011): Dopaminergic modulation of the caudal photoreceptor in crayfish. Synapse 65, 497-504 https://doi.org/10.1002/syn.20866

Rodríguez-Sosa L, Calderón-Rosete G, Anaya V, Flores G (2012): The caudal photoreceptor in crayfish: an overview. In: Photoreceptors: Physiology, Types and Abnormalities. (Eds. Akutagawa E. and Ozaki K.), pp. 59-78, Nova Science Publishers, Inc, N.Y.

Rodríguez-Sosa L, Calderón-Rosete G, Ortega-Cambranis A, DeMiguel FF (2017): Octopamine cyclic release and its modulation of visual sensitivity in crayfish. Comp. Biochem. Physiol., Part A Mol. Integr. Physiol. 203, 83-90 https://doi.org/10.1016/j.cbpa.2016.08.032

Simon WT, Edwards DH (1990): The caudal photoreceptor: a multifunctional sensory neuron may select its outputs by spike frequency. In: Frontiers in Crustacean Neurobiology. Advances in Life Sciences. (Eds. Wiese K, Krenz WD, Tautz J, Reichert H, Mulloney B), pp. 338-344, Birkhauser Verlag, N.Y. https://doi.org/10.1007/978-3-0348-5689-8_40

Society for Neuroscience policies on the use of animals and humans in research (2017): https://www.sfn.org/advocacy/policy-positions/policies-on-the-use-of-animals-and-humans-in-research

Sullivan JM, Genco MC, Marlow ED, Benton JL, Beltz BS, Sandeman DC (2009): Brain photoreceptor pathways contributing to circadian rhythmicity in crayfish. Chronobiol. Int. 26, $1136-1168$ https://doi.org/10.3109/07420520903217960 
Strauss J, Dircksen H (2010): Circadian clocks in crustaceans: identified neuronal and cellular systems. Front. Biosci. (Landmark Ed). 15, 1040-1074 https://doi.org/10.2741/3661

Tautz J, Plummer MR (1994): Comparison of directional selectivity in identified spiking and nonspiking mechanosensory neurons in the crayfish Orconectes limosus. Proc. Natl. Acad. Sci. USA 91, 5853-5857 https://doi.org/10.1073/pnas.91.13.5853

Takeuchi Y, Tobo S, Hori M (2008): Morphological asymmetry of the abdomen and behavioral laterality in atyid shrimps. Zool. Sci. 25, 355-363 https://doi.org/10.2108/zsj.25.355

Tobo S, Takeuchi Y, Hori M (2012): Morphological asymmetry and behavioral laterality in the crayfish, Procambarus clarkii. Ecol. Res. 27, 53-59 https://doi.org/10.1007/s11284-011-0867-7

Uchizono K (1962): The structure of possible photoreceptive elements in the sixth abdominal ganglion of the crayfish. J. Cell Biol. 15, 151- 154 https://doi.org/10.1083/jcb.15.1.151

Van Harreveld A (1936): A physiological solution for fresh water crustaceans. Proc. Soc. Exp. Biol. Med. 34, 428-432 https://doi.org/10.3181/00379727-34-8647C
Villagran-Vargas E, Rodríguez-Sosa L, Hustert R, Blicher A, Laub K, Heimburg T (2013): Variations in interpulse interval of double action potentials during propagation in single neurons. Synapse 67, 68-78

https://doi.org/10.1002/syn.21616

Westhoff JT, Rosenberger AE (2016): A global review of freshwater crayfish temperature tolerance, preference, and optimal growth. Rev. Fish Biol. Fisheries 26, 329-349 https://doi.org/10.1007/s11160-016-9430-5

Wilkens LA, Larimer JL (1972): The CNS photoreceptor of crayfish: morphology and synaptic activity. J. Comp. Physiol. 80, 389-407 https://doi.org/10.1007/BF00696436

Wilkens LA, Larimer JL (1976): Photosensitivity in the 6th abdominal ganglion of decapod crustaceans: A comparative study. J. Comp. Physiol. A. 106, 69-75 https://doi.org/10.1007/BF00606572

Wilkens LA (1988): The crayfish caudal photoreceptor: advances and questions after the first half century. J. Comp. Physiol. 91, 61-68 https://doi.org/10.1016/0742-8413(88)90169-7

Received: May 13, 2017

Final version accepted: August 26, 2017 